\title{
Analyse d'une année scolaire d'apprentissage du français dans une école de filles en Suède à la veille du mouvement de la Réforme
}

Elisabet Hammar

\section{CpenEdition}

\section{Journals}

Electronic version

URL: https://journals.openedition.org/dhfles/3041

DOI: $10.4000 /$ dhfles.3041

ISSN: 2221-4038

\section{Publisher}

Société Internationale pour l'Histoire du Français Langue Étrangère ou Seconde

\section{Printed version}

Date of publication: 1 June 1999

Number of pages: p.141-167

ISSN: 0992-7654

\section{Electronic reference}

Elisabet Hammar, "Analyse d'une année scolaire d'apprentissage du français dans une école de filles en Suède à la veille du mouvement de la Réforme", Documents pour l'histoire du français langue

étrangère ou seconde [Online], 23 | 1999, Online since 25 March 2015, connection on 10 March 2023

URL: http://journals.openedition.org/dhfles/3041 ; DOI: https://doi.org/10.4000/dhfles.3041

This text was automatically generated on 10 March 2023.

All rights reserved 


\title{
Analyse d'une année scolaire d'apprentissage $d u$ français dans une école de filles en Suède à la veille du mouvement de la Réforme
}

\author{
Elisabet Hammar
}

1 Dans un autre colloque SIHFLES, celui d'Edimbourg, j'ai essayé de tracer une image de l'apprentissage du français dans une école publique suédoise au cours du $19^{\mathrm{e}}$ siècle, le lycée de Stockholm¹. A cette occasion, je me suis basée sur des recherches que j'avais faites plus tôt, mais que je n'avais pas pu publier, des recherches portant sur une comparaison entre la didactique utilisée dans les écoles de garçons et celle des écoles de filles. Cette comparaison s'impose, car il est évident que les traditions pour apprendre aux filles la langue française n'ont pas été rompues par le seul fait que les circonstances de l'apprentissage avaient changé et que, désormais, les filles aussi commençaient à être "institutionnalisées ". L'intérêt d'une telle comparaison repose non seulement sur la revendication d'une histoire totale, où la vie de toute la société, avec toutes ses histoires parallèles, a le droit de figurer, mais aussi sur l'hypothèse que, dans ce domaine précis, les expériences et le savoir des femmes ont fini par s'imposer sans que les femmes aient eu le mérite, d'ailleurs, ni de les avoir fait valoir, ni, peutêtre, d'avoir donné l'exemple.

2 Au cours des recherches mentionnées ci-dessus, j'ai donc fait une autre étude, celle-ci sur une école de filles, elle aussi située dans la capitale de la Suède. C'était la plus importante école de filles de la capitale, donc la plus naturelle à comparer au lycée de Stockholm. Cette étude, je ne l'ai pourtant pas faite sur une ligne longitudinale, du point de vue temporel, mais selon une coupe transversale : l'année scolaire 1880/81. Le choix de l'année a été fait en fonction de sa place stratégique par rapport au mouvement de la Réforme, dont le point de départ consacré est le manifeste de Viëtor, en $1882^{2}$. Cette coupe m'oblige pourtant à remonter quelque peu en arrière pour situer le contexte de chaque classe. L'avantage de cette démarche par rapport à celle que j'ai 
utilisée pour le lycée de Stockholm - un exposé chronologique sur quatre-vingts ans est bien évidemment de permettre de dresser un tableau de l'instant sur toute l'étendue du corps des élèves et des enseignants. On verra qu'un tableau de l'instant porte quand même les marques de l'histoire antérieure.

\section{L'école d'Àhlin}

3 L'école d'Àhlin, fondée par Mademoiselle Karin Âhlin en 1847, a connu un grand essor vers la fin des années soixante ${ }^{3}$. Depuis 1876, l'Etat subventionnait les écoles de filles à condition qu'elles suivent certaines directives, surtout en ce qui concerne les comptes rendus ${ }^{4}$, permettant aux autorités de surveiller leurs activités. Ceci a sans doute rapproché le cursus de certaines écoles féminines de celui des lycées, tout particulièrement de la part de celles qui espéraient pouvoir, du moins plus tard, préparer leurs élèves au baccalauréat, instauré pour les lycées en 1862. L'école d'Âhlin avait cette ambition et a obtenu ce droit en $1894^{5}$, donc moins de quinze ans après Tannée de cette étude. Il me semble donc naturel de supposer que cette école ressemblera aux lycées un peu plus que certaines autres écoles de filles dans le pays.

Pour réaliser mon étude, je me suis servie non seulement des comptes rendus imprimés de l'école ${ }^{6}$, mais aussi de la matière inédite qui se trouve aux archives de la ville de Stockholm et qui consiste en registres d'élèves, " livres de cours ", projets de cours et de leçons et les notes de Karin Âhlin lors de ses inspections dans les classes.

5 Comme dans $80 \%$ de toutes les écoles de filles en Suède à l'époque, le français était la première langue étrangère ${ }^{8}$. Dans les lycées de garçons, on commençait par l'allemand et la plupart des élèves avaient aussi fait du latin avant d'entamer les études de français. Pour apprendre le français, les filles étaient donc non seulement plus jeunes que les garçons, mais aussi sans expérience d'un apprentissage d'une langue étrangère. Au début, dans l'école qui nous concerne, elles avaient environ huit ans et elles allaient poursuivre ces études pendant neuf ans, au rythme de trois à quatre heures de cours par semaine. On semble avoir eu un système souple en ce qui concerne le nombre d'heures par semaine ; les élèves faibles ont droit à plus de soutien que les autres. Une chose intéressante et dont je n'ai jamais vu l'équivalent dans un lycée, c'est qu'il n'est pas rare, dans ces classes de français, d'avoir deux, ou même trois ou quatre, enseignants de français pendant la même année scolaire, pas simultanément, mais à raison d'une heure pour l'un, et d'une heure toutes les deux semaines pour l'autre. Dans les premières classes, ces enseignants étaient des enseignantes, mais dès la troisième année, une partie de l'enseignement était assurée par un Français et, pendant les dernières années, des professeurs de lycée, donc des hommes, étaient responsables surtout du côté formel de la langue, la grammaire.

\section{Les manuels de base}

6 Trois ans plus tôt, l'école avait changé de manuel élémentaire, passant du Praktisk làrobok i franska spràket de Franz Ahn à Elementarbok i franska spràket de Cari Ploetz ${ }^{10}$. Surtout parce qu'il s'agit ici d'enfants relativement jeunes, il est difficile de comprendre cette substitution. Le manuel d'Ahn se passe le plus souvent de règles grammaticales, mais les structures nécessaires sont présentées de manières assez transparentes pour que même un enfant puisse en tirer les bonnes conclusions. L'idée 
révolutionnaire pour un novice suédophone, par exemple, selon laquelle les adjectifs ont des formes différentes s'ils qualifient un homme ou une femme, que la forme définie s'exprime par un mot détaché placé devant le mot en question, et pas comme un suffixe, et que les objets, chose bizarre, sont traités comme s'ils étaient des hommes et des femmes, est avancée très simplement par une juxtaposition transparente et par le choix de substantifs. Les déclinaisons brillent par leur absence, le vocabulaire présenté appartient au monde que l'enfant connaît bien, les phrases sont simples, même du type que l'enfant, à la rigueur, pourrait avoir envie de dire, comme Ce cheval-ci est plus petit que celui-là, mais celui-ci est plus fort. Les écoles privées et les écoles de filles, moins centrées sur l'éducation formelle des enfants, avaient été promptes à accepter et à garder ce genre de manuel, tandis que les manuels de Ploetz, une variante plus formelle que ceux d'Ahn, se sont implantés très tard à l'intérieur de l'école publique ${ }^{11}$.

7 Les élèves qui commençaient cette année-là leur quatrième année de français, connaissaient donc, sans savoir comment, tous les articles, les "déclinaisons » sans avoir appris le concept de "déclinaisons ", la formation du féminin et du pluriel des noms et des adjectifs, la comparaison des adjectifs, les pronoms possessifs et démonstratifs, l'essentiel des pronoms relatifs et interrogatifs, les numéraux, les constructions interrogatives, les négations, toutes les formes des verbes avoir et être, sauf le subjonctif, et le présent, le passé composé et l'imparfait des verbes réguliers de la première conjugaison. Non seulement elles avaient appris ces structures d'une façon plus appropriée aux enfants - je compare toujours avec le manuel de Ploetz -, avec un bagage de mots plus central, mais elles avaient avancé plus vite dans les mystères de la grammaire française.

8 L'idée fondamentale pour les deux manuels est pourtant la même, basée essentiellement sur la méthode de Seidenstticker ${ }^{12}$. Il s'agit d'une initiation scrupuleusement progressive dans les structures et le vocabulaire de la langue étrangère, divisée en leçons, qui consistent généralement en une nouvelle " règle » et quelques nouveaux mots, puis des phrases simples, sans contexte, qui incorporent ces nouvelles connaissances dans ce qu'on a appris avant, puis des phrases correspondantes en langue maternelle à traduire dans la langue étrangère. Dans le manuel de Ploetz, l'initiation à la prononciation suit le même ordre rigoureusement progressif, tandis que chez Ahn, la responsabilité de la prononciation repose sur l'enseignant, la méthodologie envisagée étant sans doute la simple imitation. Ahn n'attend pas non plus pour introduire les sons « difficiles », comme les sons nasaux et la chuintante et le $s$ sonores, dont on voit des exemples dans les toutes premières pages.

\section{Première année}

9 Les débutantes de l'école d'Âhlin de l'année en question suivent donc, pendant trois ans, le manuel de Ploetz. La progression, déjà lente dans le manuel (91 petites leçons sur environ 100 pages), frappe par sa prudence : pendant les trois premières années, on termine 15 nouvelles leçons par an, avec la révision, bien sûr, de toutes les leçons qui précèdent. Et il semble que l'on ne fasse même pas tout : la traduction des phrases suédoises en français peut ne pas avoir été entamée au cours de la première année, par exemple, car c'est pendant l'année suivante qu'on dit avoir attaqué ces traductions, l'année d'après avec un décalage correspondant : on fait, grossièrement, les traductions qui appartiennent aux leçons de l'année précédente. Cette lenteur me paraît pourtant 
si exagérée - pour la première année, ils avaient passé environ cent heures de cours à travailler treize petites pages - que je suis tentée de croire qu'elle cache quelque chose. L'expérience au cours de mes recherches sur le lycée de Stockholm, où j'avais accès, pour certaines années, à des séries doubles de comptes rendus, l'une imprimée, l'autre manuscrite ${ }^{13}$, m'a appris que l'on dit rarement tout dans les rapports annuels. Dans ce cas-ci, puisque les phrases suédoises sont si liées même à la compréhension du sens de la règle grammaticale présentée dans la leçon, il me semble possible que les phrases suédoises étaient bel et bien traduites, mais pas écrites, et donc pas dignes d'être signalées. Les enseignantes étant des femmes, et donc pas enfermées dans le formalisme, certaines lacunes visibles dans la progression dans les leçons et des preuves ultérieures que l'on ne respecte pas entièrement le plan élabore par le manuel, m'amènent à penser que ces institutrices pouvaient ajouter un grand nombre de choses, comme des jeux et des chansons, par exemple, sans pour autant vouloir l'avouer ouvertement.

Sinon, ce que les enfants avaient appris à la fin de la première année se limitait à environ 225 mots, la plupart des substantifs, la connaissance que le français a deux genres et que le pluriel donne un -s final, le présent, l'imparfait et le passé simple du verbe avoir et les pronoms démonstratifs adjectivaux. Ils avaient surtout travaillé la prononciation, ses règles, et, je suppose, aussi sa pratique, mais il y a des lacunes qui vous rendent confus : sauf dans la constellation de j'ai, j'avais, j'ai eu, ils n'avaient jamais rencontré un mot contenant la chuintante sonore, et surtout pas l'explication de ce son étranger, ni le $l l$ ou le $n g$ mouillé. Au bout d'une année de français, les enfants pouvaient donc lire et dire Samedi j'ai vu une zibeline et un renne et Nous avons fait cent prisonniers dans ce combat, mais ils seraient, d'après l'hypothèse d'un strict respect de la progression de Ploetz, incapables de lire des mots comme jardin, gens, soleil et montagne. Cette progression voulue en ce qui concerne la prononciation est peut-être à l'origine du choix un peu bizarre du vocabulaire ; éviter certains sons de façon systématique n'est pas facile. Puisque l'auteur du manuel n'a voulu expliquer que dans la quatrième leçon que les consonnes finales sont muettes, les 44 mots que les enfants ont appris dans les trois premières leçons n'ont pas de consonne finale. Avec « l'interdiction » de la chuintante sonore, des $l l$ et $n g$ mouillés, et d'autres phénomènes de prononciation pas encore expliqués, les enfants devaient apprendre, parmi les premières dizaines de mots français, le pari, la flotte et le favori.

11 Le bilan de grammaire explicite paraît aussi maigre. Mais comme cela ressort des exemples cités, Ploetz a introduit le passé composé d'autres verbes qu'avoir. Il l'a fait en se servant, pour une fois, de la méthode « intuitive » d'Ahn, en donnant toute une série de verbes au participe passé, avec leur traduction tout simplement, et pas d'explication grammaticale. Cette stratégie donne décidément une plus grande liberté et permet même d'utiliser des verbes irréguliers, comme, nous l'avons vu ci-dessus, vu et fait, mais aussi pris, bu, commis, etc. Pour le reste, on peut sentir une certaine gêne, puisque l'auteur est pris au piège entre l'esprit de système qui le porte, par exemple, à présenter toutes les formes du présent, de l'imparfait et du passé composé du verbe avoir, et l'ambition de toujours donner des exemples et un nombre suffisant d'exercices de ce système, ce qui est l'essence de cette méthode " pratique »; il ne réussit pas sur toute la ligne. 


\section{Deuxième année}

12 Au cours de la deuxième année de français, on révise les premières leçons, puis on continue encore une quinzaine de leçons, où, enfin, on apprend les chuintantes sourde et sonore, mais toujours pas les $l l$ et $n g$ mouillés ; en grammaire, le futur et le conditionnel du verbe avoir, le présent, l'imparfait et le passé composé du verbe être, la formation du féminin et du pluriel pour les adjectifs, le pronom possessif et finalement, dans les dernières leçons de l'année, numéros 29 et 30, les déclinaisons. Pour les exercices, les élèves commencent donc à traduire par écrit les phrases suédoises de l'année précédente. Elles écrivent aussi, selon l'expression énigmatique du compte rendu, « les verbes [avoir \& être] d'après le livre $~^{14}$. Mon hypothèse est que les élèves copiaient les paradigmes à partir d'un livre où les différents temps se trouvaient réunis, et pas comme dans le manuel de Ploetz, où ils étaient disposés à plusieurs endroits. Cela permettrait aussi de juxtaposer les temps des deux verbes auxiliaires et de se faire une idée de leurs ressemblances.

13 Les déclinaisons, donc, cette habitude coriace de présenter le nom français comme s'il avait des cas, comme en latin. Le fait qu'elles viennent si tard est quand même un signe qu'elles étaient en voie de disparition. Une indication est que Ploetz met à l'arrièreplan le schéma latin en utilisant surtout les termes sujet, régime direct et indirect ; les cas latins sont réduits à des initiales et l'ablatif et le vocatif ont disparu entièrement. En revanche, il n'ose pas toucher l'ordre ; l'accusatif, qui serait plus logiquement mis avec le sujet, vient en dernier lieu, après les « régimes indirects » du génitif et du datif. Un garçon, qui avait déjà vu les déclinaisons latines, s'y serait reconnu, mais dans l'école qui nous occupe, les petites filles de neuf ans devraient regarder ces schémas comme une autre preuve de l'impossibilité de comprendre le monde adulte.

\section{Troisième année}

Pour l'une des classes parallèles de la troisième année de français, sans doute celle qui avait montré les meilleurs résultats, une nouveauté bouleverse la progression de Ploetz, si elle n'était pas déjà bouleversée par les enseignantes : pendant une heure toutes les deux semaines, les petites filles de dix ans sont prises en charge par un Français, Monsieur A. Bérard. Depuis l'année précédente, ce Français enseignait dans toutes les classes à partir de la quatrième année, et donc même pour cette classe privilégiée de troisième année. Avant cette date, il ne venait que dans les plus hautes classes. Ces premières années, de la troisième à la cinquième année, ces activités ne sont décrites que comme "exercices de lecture et de conversation $»^{15}$, et il est à supposer qu'il s'agit de correction phonétique et d'un échange modeste de questions et de réponses.

Qu'est-ce que les élèves apprennent dans le manuel de Ploetz cette année-là et est-ce toujours le seul manuel ? Cette fois, la " petite grammaire » d'Emanuel Mathias Olde ${ }^{16}$ est citée comme manuel et c'est sans doute à partir de cet aperçu de la morphologie française que les élèves écrivaient, celles de la deuxième année aussi, " les verbes d'après le livre ». Cette année, hormis les verbes auxiliaires, elles écrivent aussi les paradigmes de blesser, donc un verbe régulier de la première conjugaison. 
16 Dans Ploetz, ça avance, mais comme d'habitude, ça ne va pas vite. Seulement encore une quarantaine de nouveaux mots sont appris dans les quinze nouvelles leçons, mais pour le système verbal, c'est le feu d'artifice : les verbes auxiliaires jusqu'aux paroxysmes comme que vous eussiez eu, etc., et le tout en formes interrogatives et négatives. Grâce à ces nouvelles connaissances et quelques pronoms interrogatifs, le manuel peut embarquer sur une nouveauté, même deux : les phrases françaises se développent en de petits dialogues et toutes les trois leçons sont désormais suivies d'un « questionnaire ». Le premier dialogue se déroule de la manière suivante :

17 Lektion 41. (Petit dialogue)

A. Est-il vrai, mes amis, que vous ayez été à Paris l'été dernier ? B. Oui, monsieur, cela est vrai ; nous avons été dans cette ville pendant un mois. A. Avez-vous vu les catacombes de Paris ? B. Non, monsieur ; nous n'avons pas vu ces souterrains merveilleux, car aujourd'hui les catacombes ne sont plus ouvertes aux étrangers ; mais nous avons vu les églises, les palais et les jardins publics de cette grande ville. A. Avez-vous été au spectacle ? B. Oui, monsieur ; nous avons été dans plusieurs théâtres. Vous serez peut-être étonné, quand vous aurez appris qu'à Paris il y a quatorze grands théâtres et une quantité de petits. (Ploetz $1870: 41$ ).

18 Les questionnaires commencent, il est naturel, par le plus simple : le genre de questions où l'élève ajoute très peu, un nom, un chiffre, oui/non en répétant les termes de la question, éventuellement en changeant le pronom personnel avec le verbe ou en ajoutant une négation :

Qui a découvert l'Amérique?

Où sont situées les villes de Vienne et de Saint-Pétersbourg ? Combien de leçons de français avez-vous par semaine?

As-tu été à la campagne cet été ? (Ploetz 1870 : 40).

19 Ces petites filles qui avaient suivi le manuel de Ploetz pendant trois ans ont continué encore avec ce livre pendant l'année de 1881/82. Elles ont eu droit, non seulement à un peu plus de grammaire (les pronoms relatifs et démonstratifs, la comparaison des adjectifs, tout, la formation irrégulière du pluriel, les numéraux, l'article partitif et quelques temps des autres conjugaisons régulières), mais aussi au livre de lecture à la fin du manuel, une petite vingtaine de pages de fables, de petits récits ou anecdotes. Aucune des classes que j'ai suivies, pourtant, ne finissent le cours de grammaire dans le manuel de Ploetz, qui se termine par le passif, les pronoms personnels au cas régime - il est vrai que ces pronoms sont très difficiles pour un Suédois, mais comment est-il possible d'attendre si longtemps avant de les expliquer ? - et quelques verbes irréguliers. La grammaire, désormais, est étudiée dans la « petite " ou la " grande » grammaire d'olde ${ }^{17}$.

\section{Quatrième année}

20 Les petites filles de la quatrième année, alors âgées d'environ douze ans, n'avaient donc pas suivi le régime de Ploetz, mais la progression moins sévère du manuel d'Ahn. Il est pourtant intéressant de noter que les deux progressions, degré par degré, idée, nous l'avons vu, héritée de Seidenstiicker, sont mises hors jeu dans l'école d'Âhlin par la lecture précoce - ou Ahn et Ploetz avaient-ils envisagé ce saut dans le manuel ? -des textes continus à la fin du livre, au cours de cette dernière année en tête-à-tête avec ces deux auteurs. Ces textes ne sont apparemment pas adaptés au niveau des élèves, et surtout pas au niveau où elles se trouvaient cette année-là, et l'année précédente, en ce 
qui concerne les « élèves Ahn ». Voici ce que rencontrent, dans les premiers textes, les élèves qui n'avaient pas encore, par exemple, entendu parler des pronoms personnels au cas régime et certainement pas de l'adverbe pronominal $y$, ni des verbes irréguliers, sauf avoir et être, ni même d'autres conjugaisons que la première :

Un chevreau avait grimpé sur le toit de son étable. Ayant aperçu un loup qui passait à une petite distance, il lui dit des injures. - Ce n'est pas vous, répondit le loup, qui m'injuriez, c'est le toit. (Ploetz).

Une demoiselle, nommée Caroline, alla se promener un jour sur le bord d'un ruisseau. Elle y rencontra quelques méchants enfants qui voulaient noyer un petit chien ; elle eut pitié de la pauvre bête, l'acheta et l'emporta avec elle au château. (Ahn).

21 Non seulement les petites filles avaient régulièrement, à partir de cette année, recours à un "vrai " Français, mais les enseignantes elles-mêmes quittaient, cette fois-ci explicitement, le chemin tracé. Malheureusement, nous ne pourrons sans doute jamais savoir comment cette lecture se déroulait, si les difficultés dans le texte étaient systématiquement expliquées ou si elles étaient passées sous silence. Les élèves Ahn avaient déjà lu huit pages de ce livre de lecture au cours de l'année précédente et il me semble qu'il s'agit d'une faute d'impression dans le compte rendu que, cette année, elles n'en lisent que trois, en finissant au beau milieu du récit le plus long et le plus passionnant du livre, «La belle et la bête ». L'année d'après, ce manuel ne figure plus sur la liste et les pauvres petites filles n'en auraient pas pu connaitre la fin!

Si le Français, M. Bérard, sentait le besoin d'un appui imprimé pour ses exercices de conversation, le manuel d'Ahn pouvait lui fournir un peu de matière dans ce qu'on appelle " Mots de la vie quotidienne » et "Scènes de société " ${ }^{18}$. Les " mots " réunis sous la première rubrique pouvaient être :

Grâce à Dieu ! à Dieu ne plaise ! plût à Dieu ! à la bonne heure, tout à l'heure, à cette heure, à l'instant, à demain, au plus vite, au plus tôt, au plus tard, tout au plus, tout à fait, tout à coup, à tout propos, mal à propos, à propos de rien, à peu près, etc.

Sous la deuxième rubrique, nous retrouvons les dialogues, avec variantes, du siècle précédent :

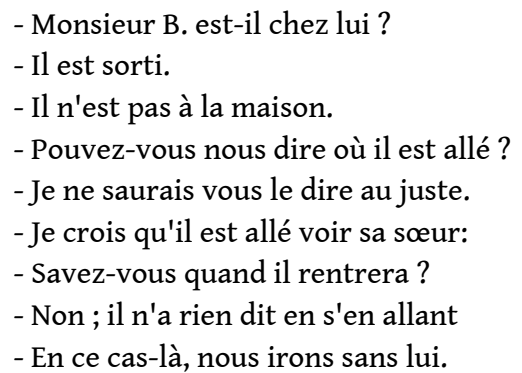

Pour la grammaire, ces élèves, déjà mieux préparées, couvrent considérablement plus que ce que .leurs cadettes apprendront clans Ploetz l'année d'après, notamment les temps principaux des deuxième et troisième conjugaisons, les complications orthographiques de la première, le reste des pronoms personnels et en et $y$. Elles continuent aussi l'étude systématique dans la " petite " grammaire d'olde, chapitre par chapitre. Ce petit livre de:50 pages est une perle de clarté, sans pour autant manquer d'explications nécessaires pour un débutant. Une telle remarque indispensable est: « Le genre des mots français n'est pas toujours le même qu'en suédois ${ }^{19}$, éclaircissement essentiel, fourni ni par Ahn, ni par Ploetz, pour un peuple qui dit, par exemple, « elle » pour le soleil et « il » pour la lune, dès qu'on ne les traite pas comme des neutres ${ }^{20}$. Le 
format d'un si petit livre ne permet naturellement que le principal, et hormis les mots ou les phrases modèles, il n'y a pas d'exemples.

Les exercices écrits se font toujours dans le cadre du manuel principal, organisés de la même manière que ceux de Ploetz, comme un renforcement des structures grammaticales apprises. La plus grande différence entre les deux est le ton; les phrases d'Àhn impliquent plus souvent les personnes je/nous et $t u / v o u s$ et tournent autour du monde enfantin, comme :

[...] Har du sert min hund ? Den är stôrre an din. Min kusin har ocksâ en hund, hwilken är mycket trogen. [...] Din lilla broder är mycket förstândig ; han är förstândigare än wâr. Wi ha en faster, hwilken är mycket rik. ${ }^{21}$ (Ahn, $4^{\mathrm{e}}$ exercice " suédois », sur l'adjectif et sa comparaison).

\section{Cinquième année}

Les élèves du niveau supérieur à celui dont je viens de rendre compte, avaient suivi à peu près le même cours. C'est une année de réorientation et de consolidation. On quitte le manuel élémentaire, mais on s'assure aussi que personne n'est perdu en cours de route ; c'est l'année où il est le plus clair que les classes parallèles sont divisées d'après les connaissances obtenues et qu'on donne aux plus faibles l'occasion de se rattraper. On pousse jusqu'à cinq heures par semaine pour l'une des classes et il est tout à fait clair que c'est la plus faible. Cette classe fait un pensum moins important dans tout, grammaire, exercices écrits et textes, malgré un nombre de cours plus généreux. Une partie des exercices verbaux est sans doute tirée de la " grande " grammaire d'olde, pour le reste on finit la « petite » au cours de l'année.

Comme on laisse de côté les manuels élémentaires, il faut un autre manuel pour les exercices écrits, qui prennent de plus en plus de place dans l'apprentissage de la langue. On choisit Franska skrifôfningar de Cari Gustaf Pauli ${ }^{22}$, adapté aux deux grammaires d'olde. On y travaille une heure par semaine et c'est un vrai travail cérébral, qui ne semble pas adapté à " l'entendement faible » des cerveaux féminins :

Ai-je ? As-tu eu ? Àura-t-il été ?[...] N'auront-ils pas ? A-t-il été ? N'aviez-vous pas eu

? Ne serais-tu pas ? Ne fûmes-nous pas ? N'auras-tu pas été ? Que nous n'eussions pas été. [...] (Pauli, $4^{e}$ éd. 1878,1: 2).

Puis suivra l'équivalent de ces phrases en suédois, à traduire en français. Pauli indique dans la préface comment il propose que les exercices se déroulent : un élève écrit une phrase au tableau et les autres le corrigent. Pour changer, l'enseignant peut travailler les exercices « cacographiques » oralement dans un groupe à côté. Ce sont des phrases françaises qui contiennent des fautes, à corriger par les élèves. Tout le manuel consiste en phrases isolées à traduire d'une langue ou d'une autre ; les numéraux se présentent en chiffres à écrire en lettres. Le dernier est 718913891 ! Cette année est aussi la première où en mentionne l'exercice de la dictée, mais sans détails.

Le texte étudié est Scènes d'enfance et de jeunesse de Madame E. de Pressensé, en une édition étrangère, puisqu'il n'a jamais été publié en Suède. Même si les jeunes filles ont étudié les textes de lecture d'Ahn, ceci est un saut en avant. Le texte qu'elles lisent cette année, "Le lundi de Pâques ", de 40 pages, donc le même texte que les jeunes Françaises de leur âge lisaient en France, commence par : 

compte rendu, on se sert du mot «traduit », mais si ce n'est pas l'enseignante qui a traduit, il est quasiment impossible à envisager que cette traduction ait été faite sur la base d'une complète compréhension grammaticale. Nous savons déjà à quel point les connaissances de la grammaire étaient modestes à ce niveau et si on compare ce qui se fait en matière de grammaire et d'exercices écrits au cours de la même année, on se demande si, avec l'abandon du manuel élémentaire, il s'est opéré une fission entre l'étude de la grammaire et celle du texte. Comme, précisément cette année, grâce à des rapports plus détaillés qu'auparavant, on peut noter une autre fission, celle qui existe entre les enseignantes principales et M. Bérard, le Français, on est tenté de croire qu'à partir de cette année, on observe une division du travail : l'apprentissage " formel ", avec la grammaire et les exercices écrits d'un côté, les « lectures et conversations : divers récits, fables, contes, anecdotes » avec un autochtone de l'autre, et la lecture et la compréhension d'un texte suivi comme un cas intermédiaire, éventuellement dirigées par une autre enseignante que celle qui est responsable de la grammaire.

\section{Sixième année}

Cette hypothèse d'une division $d u$ travail devient une certitude pour le niveau supérieur. A ce niveau, un professeur de lycée, Alfred Svensson, docteur ès lettres et 
futur éditeur du " Meisterschaftssystem » élaboré par l'Allemand R. Rosenthal ${ }^{24}$, est, dans l'une des classes, chargé de l'enseignement que l'on associe traditionnellement à l'apprentissage des langues modernes par les garçons : la grammaire et l'application de la grammaire par les exercices écrits, même en forme de ces redoutables thèmes faits à la maison ${ }^{25}$, dans cette école réservés à deux années consécutives seulement. Le Français n'est donc plus le seul homme qui enseigne à certaines de ces jeunes filles, et jusqu'à la fin de leur scolarité, la formation grammaticale d'une partie des élèves de l'école (au dernier niveau de toutes les élèves) sera faite par des professeurs de lycée. Même si cette formation est faite par une femme, comme dans plusieurs des cas par Anna Dahlström, une des femmes remarquables de cette époque qui n'ont pas encore trouvé leur place dans les encyclopédies, ni tenté un biographe jusqu'à maintenant ${ }^{26}$, il est clair que l'enseignement de la grammaire est assuré à part.

35 A partir de ce niveau, on entame l'étude systématique de la " grande» grammaire d'Olde. On commence tout simplement par le début et on ne fait exception à cet ordre que pour les verbes irréguliers. La première année, on parcourt donc les articles, le nom, l'adjectif, les numéraux, les pronoms personnels et possessifs. On continue les dictées et les exercices écrits de Pauli, aussi semblables à une gymnastique cérébrale que le niveau inférieur, mais deux des classes utilisent aussi les exercices beaucoup plus avancés de Philp ${ }^{27}$, où il y a même des textes suivis. C'est à propos de ce livre que je me pose la question de savoir si les jeunes filles ont tout de suite travaillé toute la grammaire d'olde, pas du commencement à la fin, mais tous les niveaux que cet auteur de grammaire propose, dans ce manuel marqué par l'expérience pédagogique et même expérimentale de son auteur. Son système, à mon avis génial et bien élaboré, est que toutes les parties du discours sont présentées à trois niveaux, permettant ainsi de parcourir dans un premier temps toute la grammaire à un niveau assez élémentaire, puis de la réviser en approfondissant ces connaissances par un niveau supérieur, puis de refaire la même chose une deuxième fois. Les exercices de Philp renvoient tous aux paragraphes d'olde $\mathrm{du}$ deuxième, même du troisième niveau, ce qui implique qu'apparemment on considère que les jeunes filles se trouvent à un niveau assez élevé du point de vue grammatical déjà au début de leur étude de cette grammaire, qui les suivra pendant les quatre années qui leur restent de leurs études de français à l'école.

Un temps considérable est donc consacré aux exercices écrits pour consolider les notions grammaticales, mais on écrit d'autres choses aussi : cette année, M. Bérard signale non seulement des exercices de lecture et de conversation, mais également des exercices écrits, notamment des reproductions de récits et de fables. L'écriture servira donc enfin d'expression libre, pas uniquement de panoplie d'exemples grammaticaux. Pour le texte étudié, Madame de Pressensé continue à raconter ses histoires, en quantité de plus en plus grande, car la classe la plus avancée en travaille cent pages.

\section{Septième année}

Les comptes rendus de ce niveau indiquent une progression systématique dans les études grammaticales et les exercices écrits. Toujours Olde, jusqu'à la syntaxe des verbes, toujours les manuels de Pauli et de Philp. Les « lectures et conversations » de M. Bérard continuent, et ses exercices écrits sont nommés cette fois : on reproduit dans les deux classes "Le soleil et le vent " et "Cendrillon", puis trois autres dans chaque classe, notamment " Le cerf », « Le pont de la veuve ", « Les deux voisins ", « Monsieur 
Barbe-bleue ", "La belle au bois dormant " et "La petite Anne ». S'agit-il d'une reproduction d'un texte lu ou entendu ? On ne le précise pas.

La grande différence pour les élèves de ce niveau par rapport à celles des deux niveaux inférieurs est le choix du texte "à traduire ». Celles-ci n'ont pas eu l'avantage du style familier et naturel de Madame de Pressensé, ni le désavantage de la formation exclusivement féminine de cette dame. Depuis deux ans, elles lisent les Premières lectures françaises de Ludvig Herrig, que C.G. Jungberg venait d'éditer en Suède ${ }^{28}$. Arrivées à ce niveau, elles en avaient travaillé trente-cinq pages, probablement parmi les quarante premières qui sont pourvues d'une liste de vocabulaire traduit. Cette quantité de pages nous paraît modeste, mais il s'agit d'une typographie extrêmement serrée, imprimée sur deux colonnes par page. La forme et le contenu des premiers textes sont du genre qu'on trouve dans les manuels depuis le $17^{\mathrm{e}}$ siècle : de petites fables, des poèmes et des historiettes, du type récompense des vertus et punition des vices, mais cette fois le sens moral n'est pas uniquement destiné à un public féminin ; plus tard des textes techniques sur, par exemple, la typographie et la machine à vapeur. Cette année, les deux classes travaillent encore 24 pages, indiquées pour l'une d'elles. Ces pages se trouvent dans la deuxième moitié de l'ouvrage et font partie d'une série de textes sur l'histoire de France, partant de L'invasion des Barbares et Le baptême de Clovis et se terminant par La prise de Paris, 1814. Les textes sont tirés d'auteurs nommés, comme, pour l'année actuelle, Sismonde de Sismondi, Ségur, Lamé, Fleury, Michelet, Porquet et Vigny.

L'historiographie française était traditionnellement considérée comme particulièrement utile pour l'apprentissage de la langue et de l'histoire en même temps, avec son style clair, souple, présentant des événements passionnants et émouvants, avec un sens moral, bien sûr. Cette classe part de «La Pucelle d'Orléans » et continue jusqu'à « Louis XVI » et a donc droit au procès de Louis XVI :

[...] Les uns votaient pour le bannissement, les autres pour la mort. Philippe-Égalité vota en ces termes : « Fidèle à mes devoirs, convaincu que tous ceux qui ont attenté et attenteront désormais à la souveraineté du peuple méritent la mort, je prononce la mort de Louis. » - «Ah! le monstre ! le scélérat ! son parent ! » s'écria-ton, de toutes parts. Ceux même qui avaient voté la mort se levèrent d'un mouvement spontané et laissèrent Philippe seul sur son banc (Herrig, $1877: 146$ ).

\section{Huitième année}

Le manuel de Herrig est bien connu des élèves de la huitième année : c'est la quatrième année qu'elles y travaillent, mais aussi la dernière. Est-il important, du point de vue méthodologique, qu'à ce niveau, la lecture de ce texte soit à la charge de professeurs de lycée, dans les deux classes? Est-il à présumer que, dès maintenant, le texte est analysé grammaticalement, utilisé comme une banque d'exemples ? Le nombre modeste de pages étudiées semble l'indiquer.

41 Les jeunes filles se trouvent d'ailleurs de plus en plus dans un monde masculin : à côté de l'omniprésent $\mathrm{M}$. Bérard, qui continue comme avant, toutes font de la grammaire (Olde), avec ses exercices écrits et oraux (Philp), et le texte avec des professeurs de lycée, et, en plus, on a appelé un maître particulier pour les dictées, tous les quinze jours, un certain 
Monsieur Kisling. Il n'y a que le « cours de révision », tous les quinze jours également, qui soit dirigé par une femme, Anna Dahlstrôm, qui se sert toujours des exercices écrits de Pauli et de Philp.

\section{Neuvième et dernière année}

Les jeunes filles de la neuvième et dernière année de français sont très soigneusement entourées de tous les enseignants les plus compétents. Les exercices oraux et écrits avec M. Bérard se font maintenant une fois par semaine au lieu d'une fois tous les quinze jours. En tout, on travaille le français quatre heures par semaine et les élèves de chacune des trois classes sont peu nombreuses, au maximum dix-huit. Anna Dahlström poursuit son cours de révision une heure par semaine et même les deux heures hebdomadaires qui restent pouvaient être divisées entre plusieurs professeurs. En grammaire, on finit Olde et pour certaines des élèves la syntaxe du verbe était nouvelle ; on a pourtant le temps de la réviser au cours de l'année. Les professeurs de lycée ainsi qu'Anna Dahlström continuent à se servir de traductions écrites et orales comme exercices de grammaire, et toujours d'après Philp. Il me semble que cette révision systématique, qui se fait depuis trois ans, est une des grandes caractéristiques de la méthodologie de cette époque, époque qui pourrait se compter, qui sait ?, en centaines d'années.

Pour les textes et la littérature, ces élèves avaient eu un autre cours que celles des niveaux immédiatement inférieurs. Celles-là avaient commencé leur véritable étude des textes, quatre ans avant, avec le même texte que ce niveau avait entamé l'année en question, notamment Scènes d'enfance et de jeunesse de Madame de Pressensé. Après deux ans de cette étude, elles avaient lu, au cours des deux années suivantes, Abdallah d'Edouard Laboulaye, un conte arabe de presque 300 pages, sur la piété, les vertus et la méchanceté, les aventures, l'amour, l'infidélité et la mort brutale, un ouvrage favori parmi les écoles privées et les écoles de filles à l'époque. Une des classes avait, en plus, étudié la pièce de théâtre, Les contes de la reine de Navarre d'Eugène Scribe, et une autre, celle de Théodore de Banville, Gringoire ${ }^{29}$. Ces jeunes filles semblent donc avoir accompli un cours de texte plus important que leurs successeurs.

Cette année, toutes les classes se servent du même manuel, Littérature française à l'usage des quatre classes supérieures de l'enseignement secondaire, publié en 1877 par Axel Henrik Klint. Une des classes le fait avec son professeur de lycée de Tannée précédente, E. Lindgren, les deux autres avec une des demoiselles Âhlin. Les précisions sur la lecture manquent dans les sources, on ne parle que de « morceaux choisis ». Klint recommande pourtant, dans sa préface, la lecture des pièces de théâtre : « Aucun genre ne fait mieux sentir les ressources de l'esprit et les émotions de l'âme ». (Klint, 1877 : préface)

Il présente donc les deux premiers actes du Bourgeois gentilhomme de Molière et, en entier, Le verre d'eau d'Eugène Scribe. Mais il donne aussi toute une série d'extraits de textes historiques, un autre genre qu'il préconise pour les études de français : Bossuet, Thiers, Thierry, Guizot, Ségur et Dumas. Les textes purement littéraires ne manquent pas, comme Vigny, Lamartine, Staël, Chateaubriand, Sévigné, Voltaire, Rousseau. Il finit par fournir de la poésie : Corneille, Racine, Chénier, Béranger et surtout Hugo. Des notices biographiques sur les auteurs terminent ce manuel qui permet de se faire une bonne connaissance de quelques chefs-d'œuvre de la littérature française. 
M. Bérard, en revanche, ce Français qui accompagne presque toutes les jeunes élèves de l'école d'Àhlin, précise finalement ses activités, à commencer par les exercices de conversation :

Conversations : Les conversations ont eu différents sujets, entre autres, l'explication des principaux proverbes, leur origine, leur application, avec accompagnement d'anecdotes s'y rattachant. (Àrsredogörelser).

48 Ces sujets frappent par leur complexité et leur niveau d'analyse. Du point de vue du contenu culturel, les proverbes sont un choix avisé, bien ancrés comme ils sont dans le patrimoine. Pour les exercices écrits, c'est lui qui dirige les dictées cette année, avec pour objectif explicite : "Les principales difficultés de la grammaire française ». Les exercices écrits libres ont peu à peu gagné en liberté, commençant par la reproduction et arrivant, du moins à ce niveau, à la rédaction. Cette année, les sujets de rédaction sont assez ambitieux :

Sujets historiques : Marie Stuart. Gustave Adolphe. Charles XII. Louis XI. Gustave

Vasa.

Sujets littéraires : Corneille. Racine. Molière. Mme de Staël.

Sujets d'imagination: Description d'un beau soir d'été en Suède.

Lettres à différentes personnes sur différents sujets. Résumé de [s] proverbes [et dictons populaires]. (Àrsredogörelser).

$$
49 * * *
$$
d'un peu plus près, ne ressemble à l'idée qu'on se fait après un regard rapide, et surtout
un regard de la fin du $20^{\mathrm{e}}$ siècle ; également, que des phrases laconiques dans un compte rendu peuvent cacher bien des choses.

51 Pour arriver au bout de mes objectifs, il m'a donc fallu, dans un premier temps, analyser de près les méthodes d'Ahn et de Ploetz, et il y a des choses qui m'ont surprise, favorablement et défavorablement ; favorablement, à quel point les deux cachent, parmi les exercices de leurs phrases isolées, des échanges de répliques, des «formules de politesse » et des dialogues entiers ; favorablement aussi le fait que la déclinaison des noms est absente chez Ahn et tardive et modifiée chez Ploetz; défavorablement, la lenteur et la prudence incroyables de la progression surtout chez Ploetz, même quand il s'agit de la prononciation. Le fait que des sons centraux dans la langue française ne sont introduits que très tard dans le manuel et celui que l'on semble pouvoir se passer de verbes réguliers et de pronoms personnels au cas régime très longtemps, invitent presque aux déviations de la progression faite par l'école en question.

Une autre conclusion donc : même des méthodes aussi rigoureuses que celle de ces deux auteurs ne sont pas respectées par les praticiens, au point qu'on ne les termine même pas, pour ne pas parler du fait qu'on ne les suit pas comme les auteurs l'avaient envisagé. L'idée même de la méthode, celle de ne rien introduire qui ne soit expliqué, a été négligée, du moins dans cette école. La grammaire d'olde non plus n'a sans doute pas été étudiée d'après le plan si bien élaboré par son auteur. Ce qui frappe aussi, c'est qu'après quatre ans d'études qui semblent, sur le papier, très lentes et prudentes, on fait un saut pour présenter aux jeunes Suédoises un texte fait pour les lectrices autochtones ; parallèlement on entame la "grande " grammaire d'olde de façon très avancée. Il me paraît naturel de supposer que l'enseignement du français et l'étude des textes à la fin des manuels Ahn/Ploetz ou d'autres matières linguistiques que les 
enseignantes ont pu introduire étaient plus importants et plus systématiques que les comptes rendus ne le laissent entendre.

On pourrait s'attendre à ce que cette école ambitieuse se soit adaptée au cursus des lycées de garçons et elle le fait effectivement : exercices écrits assidus, utilisation des manuels de Pauli et de Philp pour le faire, choix de Herrig comme texte et, surtout, présence assez massive, vers la fin de la scolarité, de professeurs de lycée. Peut-être pourrions-nous aussi mettre au compte de cette ambition les révisions constantes, signes assez sûrs d'une étude " sérieuse ». Le mirage du baccalauréat plane décidément au-dessus de ces têtes bouclées.

On défend pourtant ses bastions : la vieille tradition pour apprendre les langues tient bon. Est-ce par peur de la perdre que cette division de travail s'est opérée, les études grammaticales d'un côté et l'apprentissage plus libre de l'autre? Le choix des textes de Madame de Pressensé, de Scribe et de Laboulaye, ainsi que les cours de M. Bérard, sont des choses qui seraient inconcevables dans un lycée à l'époque, mais en ce qui concerne les textes, ils vont envahir les lycées après le mouvement de la réforme. Le lycée de Stockholm introduit, cette même année, et pour la première fois, le manuel de Ploetz ${ }^{30}$ et les lycées seront beaucoup plus prudents à accepter les idées de la réforme. Il me semble démontré, par cette étude approfondie, que non seulement ce mouvement était moins révolutionnaire pour les écoles de filles, mais aussi à quel point il était plus facile pour celles-ci d'en adopter et les idées et les manuels les plus radicaux.

\section{BIBLIOGRAPHY}

\section{Sources inédites}

Stockholms stadsarkiv (Archives de la ville de Stockholm) : C VI, C VII, E VI, E IX (Àhlinska skolan).

\section{Sources imprimées}

F. AHN (1846) : En ny praktisk method att lätt och pà kort tid lära sig franska spràket. (Titre des éditions ultérieures : Praktisk làrobok i franska spràket) Stockholm, Lundberg.

Ährsredogörelser för arbetet inom Ahlinska skolan. (Comptes rendus de l'école d'Âhlin) Kungl. Biblioteket, non catalogués.

H. CHRIST (1993) : « De Meidinger à Ploetz, en passant par Seidenstücker, Ahn et Ollendorff, ou le cheminement de la méthodologie synthétique ». Documents pour l'histoire du français langue étrangère ou seconde 12, pp. 5-10.

E. HAMMAR (1980) : L'enseignement du français en Suède jusqu'en 1807. Méthodes et manuels. Stockholm, Akademilitteratur.

E. HAMMAR (1985) : Manuels de français publiés à l'usage des Suédois de 1808 à 1905. Stockholm, Acta Bibliothecae Regiae Stockholmiensis XLIV. 
E. HAMMAR (1993) : « Des méthodes Ahn/Ploetz aux méthodes directes. Pratiques d'enseignement des langues en Suède de 1860 à 1905 ». Documents pour l'histoire du français langue étrangère ou seconde 12, pp. 11-15.

E. HAMMAR (1995) : « De Télémaque à la Réforme. L'institutionnalisation du français au gymnasium de Stockholm, 1824-1905 ». Documents pour l'histoire du français langue étrangère ou seconde (Profils d'enseignants, d'étudiants et d'institutions d'enseignement des langues vivantes de 1850 à 1950. Actes du colloque de la SIHFLES tenu à l'Université d'Edimbourg du 22 au 24 septembre 1994), 15, pp. 9-22.

E. HECKSCHER (1914) : Nâgra drag ur den svenska flickskolans historia. Stockholm, Norstedt.

L. HERRIG (1877) : Première lectures françaises. Stockholm, Norstedt.

A. H. KLINT (1877) : Littérature française à l'usage des quatre classes supérieures de l'enseignement secondaire. Stockholm, Gleerup.

G. KYLE (1972) : Svensk flickskola under 1800-talet. Dans Tvà studier i den svenska flickskolans historia. Stockholm, Ârsbôcker i svensk undervisningshistoria 128.

E. M. OLDE (1833) : Tabeller ôfver fransyska ordens bôjningsformer. Stockholm, Hörberg. (Titre des éditions ultérieures : Öfwersigt afdetfranska sprâkets formlära).

E. M. OLDE (1843) : Fransk spràklära fôr skolor och gymnasier. Stockholm, Hörberg.

C.G. PAULI (1865) : Franska skrifôfningar, uppstàllda efter Olde's « öfwersigt af del franska spràkets formlära » och «franska sprâklära ». Stockholm, Haeggström.

H.W. VON PHILP (1878) : Franska skriföfningar och temata med ordförklaringar [...]. Stockholm, Nyman.

C. J. PLOETZ (1870) : Elementarbok i franska spràket. Stockholm, Sievers.

E. DE PRESSENSÉ (1870) : Scènes d'enfance et de jeunesse. Paris.

A. SVENSSON (1884) : « Mästerskaps-systemet ». En ny metod att pà kort tid lära sig tala, skrijva och läsa ett spràk [...]. Stockholm, Fritze.

[W. VIËTOR] pseud. « Quousque Tandem » (1882) : Der Sprachunterricht muss umkehren! Leipzig, Reisland.

Undersökning af Sveriges högre flickskolor. Underdânigt utlâtande afgivet den 19 januari 1888 afutsedde komiterade (1888). Stockholm.

\section{NOTES}

1. Hammar (1995) : « De Télémaque à la Réforme. L'institutionnalisation du français au gymnasium de Stockholm, 1824-1905 ». Documents pour l'histoire du français langue étrangère ou seconde (Profils d'enseignants, d'étudiants et d'institutions d'enseignement des langues vivantes de 1850 à 1950. Actes du colloque de la SIHFLES tenu à l'Université d'Edimbourg du 22 au 24 septembre 1994), 15, pp. 9-22.

2. [W. Viëtor] pseud. «Quousque Tandem » (1882) : Der Sprachunterricht muss umkehren! Leipzig, Reisland.

3. Ebba Heckscher (1914) : Nàgra drag ur den svenska flickskolans historia, p. 111-

4. Kyle (1972) : Svensk flickskola under 1800-talet, pp. 193-194.

5. Heckscher (1914: 112). 
6. Ârsredogôrelser for arbetet inom Âhlinska skolan, Kungl. biblioteket, non catalogués.

7. En suédois : « klassbôcker », « lektionsbôcker », « kurs- och lektionsplaner » et « Karin Àhlins anteckningar angàende âhôrda lektioner », cotes C VI, C VII, E VI et E IX.

8. Undersôlcning af Sveriges hôgre flickskolor. Underdànigt utlàtande afgivet den 19 januari 1888 afutsedde komiterade. Stockholm $1888: 51$. Pour l'histoire des écoles de filles en Suède, voir, entre autres, G. Kyle (1972), op. cit.

9. Voir E. Harnmar (1985) : Manuels de français publiés à l'usage des Suédois de 1808 à 1905. Stockholm, Acta Bibliothecae Regiae Stockholmiensis XLIV, p. 33. La première édition suédoise a paru en 1846 et la cinquième et dernière en 1976. Deux éditions ont paru en Finlande, en 1855 et 1868.

10. Voir Harnmar (1985 : 150-51). Ce manuel a eu sept éditions, de 1870 à 1887.

11. Voir, entre autres, Hammar (1995), op. cit., et E. Hammar (1993) : « Des méthodes Ahn/Ploetz aux méthodes directes. Pratiques d'enseignement des langues en Suède de 1860 à 1905 ». Documents pour l'histoire du français langue étrangère ou seconde 12, pp. 11-15

12. Pour une vue sur le développement de ce genre de méthode, voir H. Christ (1993) : « De Meidinger à Ploetz, en passant par Seidenstücker, Ahn et Ollendorff, ou le cheminement de la méthodologie synthétique ». Documents pour l'histoire du français langue étrangère ou seconde 12, pp. 5-10.

13. Hammar (1995: 15 ss.).

14. En suédois : « verbskrivning efter bok».

15. En suédois « läs- och talövningar».

16. M. Olde (1833) : Ôfwersigt afdetfranska spràkets formära. $2^{\mathrm{e}}$ et $3^{\mathrm{e}}$ éditions 1855 et 1857 . Une première édition, avec un titre différent, Tabeller öfver fransyska ordens böjningsformer parut en 1833. Voir Harnmar (1985 : 127).

17. La «petite » est donc Olde (1833) et la « grande » E. M. Olde (1843) : Fransk spràklàra for skolor och gymnasier. Stockholm, Hôrberg. Dix éditions ont paru entre 1843 et 1886 . Voir Hammar (1985 :128-129).

18. En suédois « Ord ur dagliga lifwet » et « Ur sällskapslifwet ».

19. En suédois : «Ordens genus ar i fransfean ïoke «alltid detsamma som i swenskan ».

20. Notez qu'il ne s'agit pas d'un lapsus de la part des éditeurs suédois. En allemand, où le soleil et la lune ont le même genre qu'en suédois, la question est encore plus brûlante, vu sa fausse ressemblance avec le français en ce qui concerne le système de 'traiter les objets comme masculins et féminins.

21. La solution de cet exercice serait : « [...] As-tu vu mon chien ? Il est plus grand que le tien. Mon cousin a aussi un chien, qui est très fidèle. [...] Ton petit frère est très sage ; il est plus sage que le nôtre. Nous avons une tante, qui est très riche. »

22. $\mathrm{I}^{\mathrm{e}}$ édition 1865 et $4^{\mathrm{e}}$ et dernière 1878.

23. Voir E. Hammar (1980) : L'enseignement du français en Suède jusqu'en 1807. Méthodes et manuels. Stockholm, Akademilitteratur, pp. 96-104, 109-116.

24. Svensson (1884) : « Mästerskaps-systemet ». En ny metod att pà kort tid lära làra sig tala, skrifva och läsa ett spràk [...]. Stockholm, Fritze.

25. L'anecdote la plus connue à propos de ces thèmes, figure dans le livre de lecture de Ploetz sous le titre «Le grand malheur » : Faites-vous des thèmes, madame? demanda un jour un prince qui était encore enfant et un peu paresseux, à une dame de la cour qui se plaignait des malheurs de sa vie. - Non monseigneur. - Et bien, si vous ne faites pas de thèmes, madame, répliqua le petit prince, votre malheur n'est pas encore à son comble. (Ploetz, 1870 :104).

26. Un projet est en cours de faire une biographie collective de toute une série de femmes, souvent fondatrices d'écoles, actives à Stockholm entre 1880 et 1920

27. H. W. von Philp (1878) : Franska skriföfningar och temata med ordförklaringar [...] Stockholm, Nyman. 
28. Herrig (1877) : Premières lectures françaises. Stockholm, Norstedt. Selon la préface, ce livre avait été utilisé en Suède avant cette édition suédoise, au moyen de Tune des douze impressions allemandes stéréotypées.

29. Gringoire avait été publié en Suède (en 1879, peut-être trop tard pour ces élèves) dans la collection Petite bibliothèque française (voir Hammar 1985 :145), mais ni Abdallah, ni Les contes de la reine de Navarre n'avaient trouvé d'éditeur dans le pays.

30. Hammar (1995:20).

\section{AUTHOR}

\section{ELISABET HAMMAR}

Université de Linkôping 